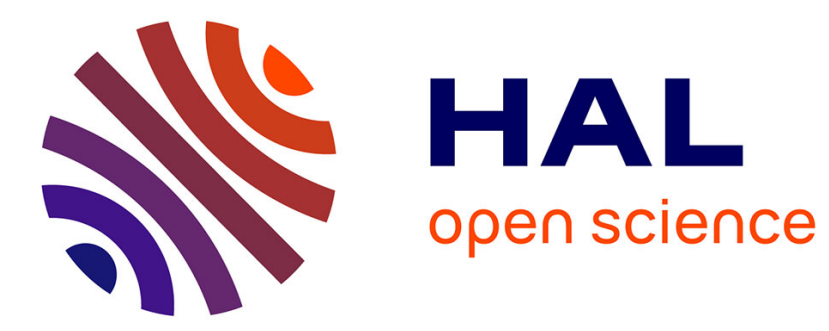

\title{
Les déterminants politiques de la fréquence des réformes démocratiques
}

Camille Bedock

\section{To cite this version:}

Camille Bedock. Les déterminants politiques de la fréquence des réformes démocratiques. Revue Française de Science Politique, 2014, 64 (5), pp.929. 10.3917/rfsp.645.0929 hal-02104976

\section{HAL Id: hal-02104976 \\ https://hal.science/hal-02104976}

Submitted on 23 Apr 2019

HAL is a multi-disciplinary open access archive for the deposit and dissemination of scientific research documents, whether they are published or not. The documents may come from teaching and research institutions in France or abroad, or from public or private research centers.
L'archive ouverte pluridisciplinaire HAL, est destinée au dépôt et à la diffusion de documents scientifiques de niveau recherche, publiés ou non, émanant des établissements d'enseignement et de recherche français ou étrangers, des laboratoires publics ou privés. 


\section{Les déterminants politiques de la fréquence des réformes démocratiques en Europe de l'Ouest, 1990-2010}

Camille Bedock

Introduction : les deux logiques de changement des institutions démocratiques

Les systèmes démocratiques sont structurés par un vaste ensemble de règles politiques formelles qui régulent la relation entre les élites au sein du système politique, les partis, et les citoyens. A ce titre, l'analyse des réformes des institutions démocratiques constitue une question centrale pour la politique comparée, plus encore dans un contexte de désenchantement envers la démocratie représentative. Pour Peter Mair, « l'intérêt intellectuel et institutionnel croissant pour la démocratie est en partie une réponse à la montée de l'indifférence populaire $»^{1}$. Dans la lignée de cette analyse, beaucoup se posent aujourd'hui la question des déterminants du recours aux réformes institutionnelles et de leur pertinence ${ }^{2}$. On peut prendre l'exemple du cas irlandais où la crise économique a donné lieu à une profonde réflexion sur le fonctionnement des institutions, et à une série d'initiatives inédites, dont l'organisation d'une convention constitutionnelle composée majoritairement de citoyens ordinaires ${ }^{3}$. Le champ de recherche portant plus spécifiquement sur les réformes électorales s'est posé déjà depuis plus d'une dizaine d'années la question des déterminants des réformes institutionnelles. Pour autant, peu d'études ont développé à ce jour une analyse du changement basée sur un vaste échantillon de réformes des institutions démocratiques, la plupart des travaux existants portant soit sur l'étude d'une seule dimension institutionnelle (le plus souvent les réformes électorales), soit sur des études de cas. Lijphart a pourtant démontré que les différentes dimensions des institutions démocratiques sont intimement liées entre elles ${ }^{4}$. A ce titre, il apparait pertinent d'étudier leurs changements en considérant aussi les réformes de manière plus systémique. Cet article s'intéresse donc simultanément à de multiples dimensions des réformes démocratiques, et se propose de répondre aux questions suivantes: peut-on expliquer pourquoi certains pays réforment davantage leurs institutions démocratiques que d'autres? Quelle logique est la mieux à même de rendre compte de la fréquence des réformes des règles démocratiques?

La réponse la plus évidente à cette question consiste à affirmer que, plus le changement est difficile, plus il est rare. Dans cette perspective, on insiste sur «la nature endogène et la construction sociale des institutions politiques $»^{5}$ et sur une approche «institutionnelle » du

\footnotetext{
${ }^{1}$ Peter Mair, Ruling the Void: The Hollowing of Western Democracy, Londres, Verso Books, 2013, p. 8.

2 Pour une synthèse de ces débats, $c$. par exemple Alan Renwick, The Politics of Electoral Reform: Changing the Rules of Democracy, Cambridge, Cambridge University Press, 2010; Bruce E. Cain, Russell J. Dalton, et Susan E. Scarrow, Democracy Transformed? Expanding Political Opportunities in Advanced Industrial Democracies, Oxford, Oxford University Press, 2003.

${ }^{3}$ Clodagh Harris, David M. Farrell, et Gemma M. Carney, "Rule by the People? Alternative Perspectives on Citizen Participation in Democratic Policymaking," Administration 60 (3), 2013, pp. 201-9; David M. Farrell, Eoin O’Malley, et Jane Suiter, "Deliberative Democracy in Action Irish-Style: The 2011 We the Citizens Pilot Citizens' Assembly," Irish Political Studies 28 (1), 2013, pp. 99-113.

${ }^{4}$ Arend Lijphart, Patterns of Democracy. Government Forms and Performance in Thirty-Six Countries, New Haven, Yale University Press, 1999.

${ }^{5}$ James March et Johan P. Olsen, "Institutional Perspectives on Political Institutions," Governance 9 (3), 1996, p. 247.
} 
changement, où le contexte politique ne joue pas un rôle central. Au contraire, ce sont les facteurs et les contraintes propres à chaque système politique qui sont mobilisées pour expliquer le changement ou la stabilité dans une perspective institutionnelle. L'idée d'une relation linéaire entre le degré de contrainte institutionnelle et la fréquence des réformes a été aussi bien avancée par des auteurs s'intéressant à la question des réformes constitutionnelles ${ }^{6}$ que par ceux qui ont développé la notion de joueurs de veto pour expliquer la stabilité des politiques publiques. ${ }^{7}$. En un mot, plus les barrières institutionnelles à l'adoption de réformes sont élevées, et plus le nombre d'acteurs en mesure de bloquer les réformes est important, moins il y aurait de réformes démocratiques adoptées.

Une théorie alternative pour analyser la fréquence des réformes, développée notamment autour de recherches sur les réformes des systèmes électoraux, consisterait au contraire à insister avant tout sur le caractère central du contexte politique pour expliquer la fréquence des réformes. Une série d'auteurs ont mis en avant l'impact sur l'adoption de réformes électorales des crises politiques majeures et de l'insatisfaction des citoyens aussi bien dans des contextes de transitions démocratiques $^{8}$ que dans des démocraties consolidées comme le Japon, l'Italie ou la NouvelleZélande. 'D'autres auteurs encore ont établi empiriquement un lien entre les mutations de la compétition politique, les transformations du système partisan et l'activisme institutionnel. Colomer et Benoit ont montré que les systèmes partisans façonnent les réformes électorales autant que l'inverse ${ }^{10}$. D'autres analyses classiques de Rokkan ou de Boix avaient déjà insisté sur l'impact de l'émergence de nouvelles forces politiques socialistes sur le passage de systèmes majoritaires à des systèmes proportionnels au début du siècle dernier ${ }^{11}$. Dans cette perspective, les réformes institutionnelles constituent des réponses à des modifications de l'environnement politique, qu'elles affectent l'opinion publique, ou l'arène électorale plus spécifiquement.

Il est par ailleurs important d'introduire, au-delà de cette distinction entre logique institutionnelle et logique politique des réformes, la distinction entre facteurs de changement à long-terme et à court-terme. Des auteurs comme Shugart ont ainsi désormais établi fermement la nécessité de distinguer les facteurs inhérents du changement et ses facteurs contingents ${ }^{12}$, ou pour le dire autrement, les facteurs mettant des systèmes donnés « en tension » et ceux qui déclenchent le changement à un moment précis. Comme chez Bawn, les institutions démocratiques sont ici envisagées comme «les produits explicites de choix sociaux » ${ }^{13}$, et donc comme le résultat de

${ }^{6}$ Donald S. Lutz, "Toward a Theory of Constitutional Amendment," The American Political Science Review 88 (2), 1994, pp. 355-70; Bjorn Erik Rasch et Roger D. Congleton, "Stability and Constitutional Amendment Procedures," dans Democratic Constitutional Design and Public Policy: Analysis and Evidence, Roger D. Congleton and Brigitta Swedenborg (ed.), Cambridge, MIT Press, 2006, pp. 319-42; Barry Weingast, "Designing Constitutional Stability," dans Democratic Constitutional Design and Public Policy: Analysis and Evidence, op. cit.

${ }^{7}$ George Tsebelis, Veto Players: How Political Institutions Work, Princeton: Princeton University Press, 2002.

${ }^{8}$ Sarah Birch et al., Embodying Democracy: Electoral System Design in Post-Communist Europe. Basingstoke, Palgrave, 2002.

${ }^{9}$ Pour une synthèse présentant les trois cas, $c f$. Alan Renwick, op.cit

${ }^{10}$ Josep Colomer, "It's Parties That Choose Electoral Systems (or, Duverger's Laws Upside Down)," Political Studies 53 (1) 2005, pp. 1-21; Kenneth Benoit, "Electoral Laws as Political Consequences: Explaining the Origins and Change of Electoral Institutions," Annual Review of Political Science no. 10, 2007, pp. 363-90.

${ }_{11}$ Stein Rokkan, Citizens, Elections, Parties. Approaches to the Comparative Study of the Processes of Development, New York: David McKay, 1970; Carles Boix, "Setting the Rules of the Game: The Choice of Electoral Systems in Advanced Democracies," American Political Science Review 93 (3), 1999, pp. 609-24.

12 Matthew S. Shugart, "Inherent and Contingent Factors in Reform Initiation in Plurality Systems," dans To Keep or to Change First Past the Post? The Politics of Electoral Reform, André Blais (ed.), Oxford, Oxford University Press, 2008, pp. 7-61.

${ }^{13}$ Kathleen Bawn, "The Logic of Institutional Preferences: German Electoral Law as a Social Choice Outcome," American Journal of Political Science 37 (4), 1993, p. 965. 
décisions des élites politiques et d'autres acteurs qui choisissent de modifier les règles formelles de la démocratie. L'argument principal développé ici consiste à montrer que les élites politiques réagissent aux changements de l'opinion publique et aux changements électoraux par le biais du changement institutionnel, et donc que la logique politique prévaut sur la logique institutionnelle. On s'attache donc à distinguer les caractéristiques durables qui ouvrent la voie aux réformes, et les chocs qui permettent d'expliquer une hausse de l'activisme institutionnel à des moments précis. Trois aspects sont analysés de plus près : l'impact du niveau de soutien politique, celui de la hausse de la volatilité électorale, et celui de l'alternance politique. L'article s'attache à démontrer qu'un faible niveau de soutien politique permet d'expliquer un recours plus fréquent aux réformes institutionnelles à long terme ; tandis qu'à court terme, l'alternance politique et la montée de l'incertitude électorale sont associées à un nombre plus important de réformes dans une législature donnée. Au contraire, l'article montre la faible pertinence des contraintes institutionnelles comme la rigidité constitutionnelle et le nombre de joueurs de veto pour comprendre la fréquence du changement.

\section{Contraintes institutionnelles, soutien politique, changement électoral et réformes des règles démocratiques}

\section{Une relation linéaire entre contraintes institutionnelles et réformes des règles démocratiques?}

Dans cet article on s'intéresse à une série de réformes démocratiques faisant l'objet de l'adoption d'une loi au niveau national. Les institutions sont typiquement caractérisées comme des «dispositions stables, valorisées et récurrentes des comportements ${ }^{14}$. Pour modifier les institutions démocratiques, un certain nombre de barrières doivent être surmontées, variables selon les systèmes politiques. Ces barrières prennent la forme de ce que Congleton appelle «les lois pour faire les lois ${ }^{15}$, ou encore de l'existence d'un certain nombre d'acteurs capables d'opposer leur veto à une réforme.

Dans une étude fondatrice sur le sujet, Lutz a démontré empiriquement qu'il existe un lien empirique d'interaction entre la difficulté d'amender la constitution, la longueur de celle-ci, et la fréquence de ses modifications ${ }^{16}$. En se basant sur ses résultats, d'autres auteurs ont montré l'importance de ce qu'ils appellent l' «effet coût» pour expliquer la fréquence des réformes constitutionnelles. Rasch et Congleton montrent ainsi que «les procédures d'amendement peuvent contribuer à la fois à la stabilité et à la durabilité d'un régime constitutionnel $»^{17}$. D'autres recherches, qui se sont penchées sur la question de la durabilité des constitutions ontelles montré qu'il existe une relation positive, mais non-linéaire, entre la rigidité constitutionnelle et la durée des constitutions. En effet, même si les deux éléments sont liés, une constitution trop rigide n'est plus susceptible d'adaptation ce qui peut conduire à des procédures de remplacement complet d'une constitution par une autre ${ }^{18}$. Ainsi, toutes ces études établissent un lien entre la difficulté de réformer les institutions, la fréquence des amendements constitutionnels, et la durée des constitutions. Si l'on appliquait ces conclusions aux réformes qui nous intéressent ici, on

\footnotetext{
14 Samuel P. Huntington, "Political Development and Political Decay," World Politics 17 (3), 1965, pp. 386430.

15 Roger D. Congleton, Improving Democracy through Constitutional Reform: Some Swedish Lessons, Norwell, Kluwer Academic Publishers, 2003, p.11.

${ }^{16}$ Lutz, "Toward a Theory of Constitutional Amendment.", op. cit.

${ }^{17}$ Rasch and Congleton, "Stability and Constitutional Amendment Procedures", op. cit. p.537.

18 Zachary Elkins, Tom Ginsburg, et James Melton, The Endurance of National Constitutions, Cambridge, Cambridge University Press, 2009.
} 
pourrait donc anticiper que la fréquence des réformes devrait être plus faible quand la procédure de réforme de la constitution est plus contraignante.

L'idée d'une relation entre contrainte institutionnelle et stabilité constitue l'argument central des travaux qui ont introduit et développé la notion de «point de veto » d'Immergut ${ }^{19}$, systématisée par l'approche en termes de joueur de veto de Tsebelis ${ }^{20}$. L'argument principal de ce dernier postule que "pour changer le statu quo législatif, un certain nombre d'acteurs individuels ou collectifs doivent donner leur accord au changement proposé $»^{21}$. Par conséquent, la capacité d'altérer le statu quo dépend du nombre de joueurs de veto et de la distance idéologique qui les sépare. Il conclut en prédisant la stabilité des politiques publiques dans les pays caractérisés par un nombre important de joueurs de veto, tandis que les pays avec un joueur de véto unique sont susceptibles de connaitre une plus grande instabilité. Dans des versions plus récentes de son argument, celui-ci se contente d'affirmer «qu'une vérité analytique liant les joueurs de véto à la stabilité des politiques est que au fur et à mesure que le nombre de joueurs de véto augmente, la stabilité des politiques ne diminue pas $»^{22}$. Si l'argument de Tsebelis s'appliquait au-delà des politiques publiques aux réformes de la démocratie, on devrait donc constater qu'il y a beaucoup plus de réformes dans des pays généralement caractérisés par le gouvernement d'un seul parti, comme le Royaume-Uni, et moins dans les pays gouvernés par de larges coalitions hétérogènes idéologiquement comme la Belgique. Bien que ces auteurs proposent une approche du changement institutionnel tout à fait distincte de celle de Tsebelis, on retrouve l'idée sous-jacente du lien entre joueurs de veto et stabilité des règles institutionnelles formelles existantes dans les travaux de Mahoney et Thelen. Ils prévoient par exemple que le changement de type " displacement», dans lequel des règles existantes sont remplacées par des nouvelles règles, est plus probable quand les possibilités de véto des acteurs sont faibles et leur marge d'interprétation des règles est limitée. Dans des circonstances où la capacité de véto est forte, d'autres types de changements des règles sont plus probables : les acteurs tendront plutôt à réinterpréter les règles existantes ("drift») ou à ajouter des nouvelles règles en sus des règles existantes (《layering $)^{23}$.

D'autres auteurs ont développé une vision moins linéaire du lien entre joueurs de véto et stabilité. Crepaz distingue l'impact, contrairement à Tsebelis, des joueurs de véto collectifs et des joueurs de véto partisans. Alors que la présence de plusieurs joueurs de véto collectifs, comme par exemple plusieurs chambres au parlement, favorise l'adoption de réformes par le biais d'un processus de «logrolling», la présence de multiples joueurs de véto partisans mène à l'inertie des politiques de redistribution ${ }^{24}$. D'autres auteurs comme Rahat qui se sont spécifiquement intéressés aux réformes de la structure des régimes démocratiques postulent même qu'il existe une relation curvilinéaire entre le nombre de joueurs de véto et de réformes ${ }^{25}$ : les réformes seraient plus faciles quand le pouvoir est très concentré, ou quand il est si dispersé qu'aucun acteur ne peut à lui seul les bloquer. Cette idée avait également été avancée par Hellman qui

${ }^{19}$ Ellen M. Immergut, "Institutions, Veto Points, and Policy Results: A Comparative Analysis of Health Care," Journal of Public Policy 10 (4), 1990, pp. 391-416; Ellen M. Immergut, Health Politics: Interests and Institutions in Western Europe, Cambridge, Cambridge University Press, 1992.

${ }^{20} \mathrm{Op}$. cit.

${ }^{21}$ Ibid., p. 2.

22 George Tsebelis, "Veto Player Theory and Policy Change: An Introduction," dans Reform Processes and Policy Change: Veto Players and Decision-Making in Modern Democracies, Thomas König, Marc Debus, et George Tsebelis (ed.), New York, Springer, 2010, p. 4.

${ }^{23}$ James Mahoney et Kathleen Thelen, "A Theory of Gradual Institutional Change," dans Explaining Institutional Change: Ambiguity, Agency and Power, James Mahoney et Kathleen Thelen (eds). Cambridge, Cambridge University Press, 2010, pp. 1-38.

${ }^{24}$ Markus M. L. Crepaz, "Global, Constitutional, and Partisan Determinants of Redistribution in Fifteen OECD Countries," Comparative Politics 34 (2), 2002, pp. 169-88.

${ }_{25}$ Gideon Rahat, The Politics of Regime Structure Reform in Democracies: Israel in Comparative and Theoretical Perspective, Albany, SUNY Press, 2008, p. 5. 
montrait que dans le cas des pays d'Europe de l'est, un nombre élevé de joueurs de véto pouvait aussi permettre une capacité de changement plus importante dans des situations où, grâce à de larges coalitions inclusives, il est plus difficile pour chaque parti de s'opposer aux réformes ${ }^{26}$. Cependant, il est généralement attendu qu'une relation linéaire et positive existe entre le degré de contrainte institutionnelle et la difficulté de réformer.

Pour évaluer la pertinence de l'approche institutionnelle, on vérifiera donc ici l'hypothèse selon laquelle le degré de contrainte institutionnelle mesuré par la rigidité constitutionnelle et le nombre de joueurs de vétos est un frein aux réformes, en gardant en tête les réserves de plusieurs auteurs qui suggèrent que ce lien n'est pas nécessairement linéaire ou absolu.

\section{Le lien entre niveau de soutien politique et réformes des règles démocratiques}

Dalton a décrit le phénomène $\mathrm{d}^{\text {' }}$ "érosion du soutien politique » qui frappe toutes les démocraties industrielles avancées, et donc l'Europe de l'Ouest en particulier ${ }^{27}$. Les partis politiques et les institutions démocratiques font donc face à des défis sans précédent pour leur légitimité. Le niveau de soutien politique peut être, selon lui, décomposé en cinq objets distincts : la confiance envers les autorités publiques (partis et politiciens), la confiance envers les institutions politiques (gouvernement, parlement), les évaluations de la performance du régime (le niveau de satisfaction envers le fonctionnement de la démocratie), le niveau de soutien pour les principes démocratiques, et le niveau de soutien à sa communauté politique. Dalton fait le constat que «l'insatisfaction politique s'étend désormais au-delà des autorités publiques pour atteindre les institutions et les normes du processus démocratique ${ }^{28}$. Il ajoute qu' "alors que l'objet d'insatisfaction devient plus général (...) les implications politiques s'élargissent. Un déclin du soutien au processus politique pourrait provoquer un défi fondamental pour les structures constitutionnelles ou des appels à réformer les procédures de gouvernement $»^{29}$. Dans un ouvrage collectif édité par Cain, Dalton et Scarrow portant sur les transformations contemporaines de la démocratie, l'hypothèse liant le déclin du soutien politique à un recours accru aux réformes institutionnelles tend à être confirmée : les auteurs montrent un activisme institutionnel en hausse se manifestant, par exemple, par la multiplication des arènes électorales dans lesquelles peuvent s'exprimer les citoyens, l'essor des réformes de décentralisation, le développement des instances de sélection directe des candidats, la multiplication de mesures en faveur de la transparence, etc ${ }^{30}$. En un mot, l'argument central de l'ouvrage postule que «le remède pour la démocratie est plus de démocratie $»^{31}$. En découle une série des réformes institutionnelles dont le but est de favoriser la participation des citoyens au processus politique.

Norris a été l'une des premières à tenter d'établir un lien statistique entre légitimité démocratique et réforme électorale. En comparant les pays inclus dans le World Value Survey entre 1993 et 2004, elle montre qu'il existe un lien positif entre les "aspirations démocratiques » (le soutien à l'idéal démocratique) et le recours ultérieur aux réformes électorales ${ }^{32}$. Elle ne trouve

\footnotetext{
${ }^{26}$ Joel S. Hellman, "Winners Take All: The Politics of Partial Reform in Postcommunist Transitions," World Politics 50 (2), 1998, pp. 203-34.

27 Russell J. Dalton, Democratic Challenges, Democratic Choices: The Erosion of Political Support in Advanced Industrial Democracies, Oxford, Oxford University Press, 2004.

${ }^{28}$ Ibid., p.9.

${ }^{29}$ Ibid., p.7.

${ }^{30}$ Bruce E. Cain, Russell J. Dalton et Susan E. Scarrow (ed.), Democracy Transformed? Expanding Political Opportunities in Advanced Industrial Democracies, Oxford: Oxford University Press, 2003.

31 Bruce E. Cain, Russell J. Dalton et Susan E. Scarrow, "New Forms of Democracy? Reform and Transformation of Democratic Institutions," dans Democracy Transformed?, op.cit.

32 Pippa Norris, "Cultural Explanations of Electoral Reform: A Policy Cycle Model," West European Politics 34 (3), 2011, pp. 531-50.
} 
en revanche pas de relation significative entre la confiance dans les institutions, l'évaluation de la performance démocratique, et le recours aux réformes électorales. Cette recherche établit plusieurs constats importants. Le premier est l'impact de l'opinion publique et du niveau de soutien politique sur la propension à réformer. Le deuxième, qui en découle directement, est la remise en question de l'idée d'un processus de réformes institutionnelles résultant purement des interactions entre élites politiques dans lequel les citoyens ne jouent qu'un rôle accessoire. Le manque de confiance politique est ici envisagé comme un facteur inhérent de réforme qui intensifie la question de la réforme électorale et encourage la mise en œuvre de révisions.

Pour résumer, qu'ils s'intéressent aux institutions démocratiques de manière générale ou aux réformes électorales en particulier, ces auteurs postulent une relation négative entre le niveau de soutien politique et le recours aux réformes, attendu que les démocraties où ce soutien est le plus faible ont davantage tendance à réformer la démocratie.

\section{Incertitude électorale et réforme des règles démocratiques}

Le niveau de soutien politique peut être considéré comme un élément structurant de l'environnement dans lequel évoluent les partis politiques. Pour autant, si l'on s'intéresse plus spécifiquement au moment auquel les réformes ont lieu, la question des évolutions de court terme ayant lieu, notamment, dans l'arène électorale, devient centrale pour expliquer la fréquence du recours aux réformes. La littérature sur le changement des systèmes électoraux a notamment permis d'établir un lien entre incertitude électorale et recours aux réformes électorales, démontrant que les diverses fortunes électorales des partis ont un impact clair sur leur propension à soutenir le changement.

Des auteurs comme Andrews et Jackman se sont centrés sur l'impact de l'incertitude sur le comportement des acteurs politiques qui sont en mesure de mettre en œuvre les réformes, ironiquement appelés des «idiots stratégiques »" Ils montrent comment, placés dans un contexte de forte incertitude, les partis politiques sont incités à agir en suivant une logique court-termiste qui peut résulter dans des erreurs de jugement quant aux effets anticipés des nouvelles règles. Leur argument central postule que "pour que les acteurs politiques s'engagent dans la réforme des procédures avec lesquelles ils ont gagné en premier lieu, ils doivent en venir à croire soit que les arrangements existants vont avoir un effet négatif sur leurs chances de victoire, soit parce qu'ils font face à une incertitude considérable, ou les deux $»^{34}$. Leur étude montre également un lien très clair entre l'incertitude et les changements électoraux, montrant que les partis ont basé leur soutien au passage d'un système électoral majoritaire à la représentation proportionnelle sur leur propre performance électorale à l'élection la plus récente. Ainsi, les partis qui ont obtenu une très bonne performance électorale ont tendu à professer la conservation du système électoral existant, tandis que les partis qui ont subi des revers ont promu l'introduction de la représentation proportionnelle.

D'autres études ont souligné l'impact de la volatilité sur les réformes électorales. Là encore, la hausse de la volatilité est interprétée comme un indicateur du niveau d'incertitude auxquels les réformateurs (qui sont ici essentiellement les partis) doivent faire face. Remmer montre ainsi comment dans les dernières décennies en Amérique latine, les réformes électorales ont à la fois été la conséquence de la volatilité électorale tout en alimentant cette volatilité par les réajustements induits par les nouvelles règles ${ }^{35}$. Enfin, des travaux très récents portant sur 45 pays

\footnotetext{
${ }^{33}$ Josephine T. Andrews et Robert W. Jackman, "Strategic Fools: Electoral Rule Choice Under Extreme Uncertainty," Electoral Studies 24 (1), 2005, pp. 65-84.

${ }^{34}$ Ibid., p.66.

35 Karen L. Remmer, "The Politics of Institutional Change: Electoral Reform in Latin America 19782002," Party Politics 14 (1), 2008, p. 24.
} 
européens entre 1945 et 2002 et tirés du projet «Electoral Systems Changes in Europe » qui couvre de multiples dimensions du système électoral (formule électorale, mais aussi magnitude, taille de l'assemblée, type de vote, etc.) ont montré non seulement que la hausse de la volatilité augmente la probabilité de réformer le système électoral, mais aussi que l'arrivée dans l'arène électorale de nouveaux entrants pousse les partis à adopter des réformes qui rendent le système moins inclusif ${ }^{36}$. Dans cette perspective, les réformes électorales induites par la volatilité électorale sont une réponse directe à une menace électorale posée par de nouveaux partis comme Boix avait déjà pu le montrer en s'intéressant à l'impact de la montée des partis socialistes sur le passage d'un système électoral majoritaire à la représentation proportionnelle au début du $20^{\text {ème }}$ siècle en Europe de l'Ouest ${ }^{37}$.

\section{Alternance politique et réforme des règles démocratiques}

En tentant d'évaluer l'impact des évolutions électorales sur les réformes démocratiques, un certain nombre d'auteurs, souvent dans le cadre de l'étude des réformes électorales, ont démontré l'impact du succès ou de l'échec électoral sur le soutien d'un parti particulier au changement. L'impact de l'alternance politique a été interprété de deux manières. D’une part, certains auteurs ont développé l'idée que les différents partis ont des préférences distinctes quant aux réformes, certains d'entre eux étant, pour des raisons normatives ou stratégiques, plus prompts à soutenir la réforme que d'autres. D'autre part, d'autres auteurs adoptent une interprétation légèrement plus complexe en montrant l'impact du statut de parti sortant: les partis qui ont passé une longue période au pouvoir sont moins susceptibles d'avoir des préférences favorables au changement institutionnel.

La première interprétation postule donc que les partis dans l'opposition et ceux au gouvernement tendent à avoir des préférences différentes sur le système électoral idéal. Cette idée est importante dans la mesure où il est désormais bien établi que les motivations égoïstes des acteurs sont centrales pour évaluer leur position sur les réformes électorales ${ }^{38}$ dans la mesure où ceux-ci sont essentiellement «redistributifs» et impliquent nécessairement la désignation de vainqueurs et de perdants ${ }^{39}$. D'autres travaux plus récents ont cependant aussi insisté sur le fait que les positions des individus et des partis à propos des réformes électorales dépendent également largement de leurs préférences normatives sur ce qu'ils considèrent être le meilleur système, indépendamment de l'idéologie ou de la maximisation des intérêts ${ }^{40}$. S'intéressant plus spécifiquement aux réformes électorales mineures, et à partir de l'exemple des Pays-Bas, des auteurs comme Jacobs et Leyenaar ont montré que lorsque les conséquences d'une réforme

\footnotetext{
${ }^{36}$ Lidia Nunez, Pablo Simon Cosano, et Jean-Benoit Pilet, "We Don't Want You to Play With Them! Economic Crisis, Electoral Volatility and the Dynamics of Electoral Reform," dans l'atelier Economic Recession, Democratic Recession? présenté à la ECPR 42nd Joint Session of Workshops, Université de Salamanque, 2014.

37 Carles Boix, "Setting the Rules of the Game: The Choice of Electoral Systems in Advanced Democracies," American Political Science Review 93 (3), 1999, pp. 609-24.

38 Jean-Benoit Pilet, "The Future Is Imagination, the Present Is Reality: Why Do Big Ruling Parties Oppose Majority Systems? A Belgian Case Study," Representation 44 (1), 2008, pp. 41-50; Gideon Rahat, "The Study of the Politics of Electoral Reform in the 1990s: Theoretical and Methodological Lessons," Comparative Politics 36 (4), 2004, pp. 461-79; Alan Renwick, Chris Hanretty, et David Hine, "Partisan SelfInterest and Electoral Reform: The New Italian Law of 2005," Electoral Studies 28 (3), 2009, pp. 437-47.

${ }^{39}$ George Tsebelis, Nested Games: Rational Choice in Comparative Perspective, Berkeley, University of California Press, 1990.

40 Damien Bol, "Electoral Reform, Values and Party Self-Interest," Party Politics, publié en ligne le 9 décembre 2013, 2013, 1354068813511590; Alan Renwick et Jean-Benoit Pilet, "The Multiple Role of Values in Electoral Reform: Mechanisms and Hypotheses," presenté à l'Annual Meeting of the American Political Science Association, Toronto, 2009, p. 44.
} 
donnée sur la distribution du pouvoir ne sont pas claires pour les partis politiques, leur soutien ou leur opposition à cette réforme dépend essentiellement de considérations normatives sur la désirabilité de la réforme ${ }^{41}$.

La deuxième interprétation du rôle de l'alternance, qui est notamment basée sur l'étude des opinions des parlementaires sur les réformes électorales, montre un lien clair entre le fait d'être un député sortant, d'appartenir à la majorité, et une faible propension à soutenir les réformes électorales ${ }^{42}$. Ce résultat confirme des conclusions similaires portant sur le soutien des élites politiques en faveur de l'adoption de mécanismes de démocratie directe ${ }^{43}$. Dans une perspective similaire, Bol et Pilet ont analysé la position des partis dans 13 processus de réformes électoraux en mettant en évidence le fait que les partis qui avaient été au gouvernement plus de $60 \%$ du temps pendant les 25 dernières années avaient une chance significativement plus forte de soutenir le système électoral existant, quelles que soient les conséquences attendues en termes de sièges ${ }^{44}$.

La plupart des investigations empiriques se sont pourtant concentrées sur les réformes électorales, et pas sur les réformes démocratiques de façon plus générale. Or, peut-on attendre un effet similaire des contraintes institutionnelles, du soutien politique, de la volatilité et de l'alternance sur les réformes démocratiques de façon plus générale ? Il y a des raisons de penser que ces facteurs s'appliquent de manière plus forte encore pour les réformes de la démocratie. Pour des auteurs comme Colomer, changer les institutions serait même plus difficile que changer les politiques publiques. Il affirme ainsi que «les modèles standard de science politique et d'études des politiques publiques se concentrent essentiellement sur trois éléments: les préférences des citoyens, les positions des partis et des candidats, et les règles institutionnelles. L'hypothèse typique est que l'ensemble des règles institutionnelles est l'élément le plus stable $»^{45}$. Les contraintes institutionnelles devraient donc jouer un rôle important. Deuxièmement, les réformes électorales sont des exemples relativement " purs » de réformes redistributives à somme nulle, dans lesquelles les élites ont des intérêts personnels puissants. Cependant, il a déjà été démontré que ces intérêts personnels et d'autres barrières peuvent être surmontés et mener à des réformes. Les auteurs insistent de plus en plus sur le fait que, au-delà des motivations contingentes aux conséquences des réformes et basées sur l'anticipation de leurs résultats (outcomecontingent motivations), les motivations basées sur l'acte de réformer (act-contingent motivations) sont également centrales pour expliquer le soutien aux réformes électorales ${ }^{46}$. Dans le cas de réformes où les conséquences en termes de distribution du pouvoir sont plus incertaines que pour les réformes électorales, il y a de fortes raisons de penser que les réformateurs basent fortement leurs jugements sur des motivations liées à la perception de l'acte même de réformer, et sont plus sensibles aux changements de l'opinion publique, qu'elle se manifeste dans le niveau de soutien politique ou dans les urnes.

\section{Hypothèses et réformes analysées}

${ }^{41}$ Kristof Jacobs et Monique Leyenaar, "A Conceptual Framework for Major, Minor, and Technical Electoral Reform," West European Politics 34 (3) , 2011, pp. 495-513.

42 Shaun Bowler, Todd Donovan, et Jeffrey A. Karp, "Why Politicians Like Electoral Institutions: Self Interest, Values, or Ideology?," Journal of Politics 68 (2), 2006, pp. 434-46.

${ }^{43}$ Shaun Bowler, Todd Donovan, et Jeffrey A. Karp, "When Might Institutions Change? Elite Support for Direct Democracy in Three Nations," Political Research Quarterly 55 (4), 2002, pp. 731-54.

44 Jean-Benoit Pilet et Damien Bol, "Party Preferences and Electoral Reform: How Time in Government Affects the Likelihood of Supporting Electoral Change," West European Politics 34 (3), 2011, pp. 568-86.

${ }^{45}$ Josep Colomer, "Disequilibrium Institutions and Pluralist Democracy," Joumal of Theoretical Politics 13 (2), 2001, p.235.

${ }^{46}$ Alan Renwick, The Politics of Electoral Reform: Changing the Rules of Democracy, op. cit.; Alan Renwick, “Electoral Reform in Europe since 1945," West European Politics 34 (3), 2011, pp. 456-77. 


\section{Des facteurs distincts à court et à long-terme}

A partir des considérations théoriques précédentes, quatre hypothèses principales sont développées dans deux modèles empiriques distincts. On s'attache à démontrer qu'il est faut clairement distinguer les facteurs qui créent des conditions favorables au changement des institutions démocratiques à long-terme, et les chocs de court-terme qui permettent d'expliquer pourquoi des réformes ont lieu en nombre plus important à des moments précis.

H1. A long terme et à court terme, plus le degré de contrainte institutionnelle est fort, moins un pays réforme ses institutions.

Dans cette première hypothèse, il s'agit de tester la pertinence de la logique institutionnelle, afin d'évaluer s'il existe effectivement une relation linéaire et négative entre le degré de contrainte institutionnelle et la fréquence des réformes à long-terme comme à court-terme. En d'autres termes, il s'agit de vérifier si la logique mise en évidence par Tsebelis ou par Lutz selon laquelle un nombre plus élevé de joueurs de véto et des institutions plus rigides freinent les réformes est pertinente pour analyser la fréquence des réformes démocratiques.

H2. A long-terme, plus le niveau de soutien politique pour les institutions et le système démocratique est bas, plus un pays réforme ses institutions.

On s'attend à ce que les élites au pouvoir aient tendance à éviter de réformer les institutions démocratiques à moins qu'elles ne se sentent contraintes de le faire du fait d'un faible niveau de soutien politique, et donc d'une insatisfaction des citoyens envers leur système politique du fait de son manque de légitimité. Cette hypothèse suppose également que les élites politiques en mesure de réformer les institutions considèrent qu'un niveau minimal de soutien est nécessaire et souhaitable pour que les institutions démocratiques puissent fonctionner. Une hypothèse alternative, qui sera testée, pourrait consister à penser que les élites réagissent au niveau de soutien politique non seulement à long-terme, mais aussi à plus court-terme (et donc qu'ils perçoivent et réagissent à une baisse de confiance des citoyens envers le fonctionnement de leur démocratie et de leurs institutions).

H3. A court-terme, quand le niveau de volatilité dans l'arène électorale augmente, le nombre de réformes institutionnelles adoptées dans la législature suivante augmente.

Le niveau de volatilité électorale peut être perçu comme l'expression tangible des variations des préférences électorales d'une élection à une autre, mais aussi comme un indicateur du niveau d'incertitude électorale auquel les acteurs politiques doivent faire face. Chaque système politique est caractérisé par un niveau inhérent de volatilité qui est lié à la structure de son système partisan et au nombre de partis en compétition. Toute angmentation de ce niveau de volatilité peut être analysée comme la traduction d'une montée de l'incertitude quant à l'équilibre des pouvoirs entre les partis. Une telle montée de l'incertitude pourrait également être le résultat d'une insatisfaction croissante avec le système politique, comme l'illustrent par exemple des niveaux de volatilité record enregistrés en Italie en 1994, ou en Irlande en 2011, au lendemain d'une crise de légitimité très sévère pour les partis politiques. Dans de telles circonstances, la montée de la volatilité implique un fort niveau d'incertitude affectant l'ensemble des partis politiques. Dans un scénario comme dans l'autre, on s'attend qu'une montée du niveau de volatilité permette de prédire que plus de réformes sont adoptés dans la législature suivante. Pour vérifier que l'impact de cette variable se limite au court-terme, on testera aussi l'impact du niveau moyen de volatilité sur le nombre de réformes à long-terme.

H4. Quand des acteurs auparavant dans l'opposition arrivent au pouvoir, le nombre de réformes institutionnelles adoptées dans la législature suivante augmente.

Par définition, l'alternance politique se traduit par l'arrivée au pouvoir de forces qui étaient auparavant cantonnées dans l'opposition. On s'attend ici à ce que l'alternance constitue une forte 
incitation à l'adoption de réformes institutionnelles, en ce qu'elle donne à de nouveaux partis l'opportunité politique de conduite des réformes, capacité qu'ils n'avaient pas auparavant. On part donc du présupposé que les partis au gouvernement et les partis d'opposition ont développé, à travers le temps, des préférences distinctives en lien avec les réformes de la démocratie. On peut également supputer que lorsque les partis auparavant dans l'opposition parviennent au gouvernement, ils ont dans les mains la capacité politique de réformer un système dont ils étaient moins satisfaits que les sortants. Cette proposition est en lien avec l'idée que les partis d'opposition tendent à être plus réformistes et moins enclins à l'aversion au risque. Dans tous les cas, on attend que l'alternance amène au pouvoir des partis ayant des préférences différentes sur les institutions, et donc que plus de réformes aient lieu dans une législature donnée lorsque les sortants perdent les élections.

\section{Une analyse longitudinale et multidimensionnelle des réformes démocratiques}

Pour tester les quatre hypothèses précédentes et l'impact du degré de contrainte institutionnelle, du niveau de soutien politique, de la hausse de la volatilité et de l'alternance politique sur la fréquence des réformes institutionnelles, cet article utilise une base de données intitulée "Institutional Change in Advanced European Democracies » et développée dans le cadre du projet de recherche SIEPOL (Seclusion and Inclusion in the European Polity: Institutional Change and Democratic Practices) ${ }^{47}$. Cette base de données couvre 20 années de réformes institutionnelles (1990-2010) dans 18 pays européens qui sont devenus démocratiques avant 1989 : l'Allemagne, l'Autriche, la Belgique, le Danemark, l'Espagne, la Finlande, la France, la Grèce, l'Irlande, l'Islande, l'Italie, le Luxembourg, la Norvège, les Pays-Bas, le Portugal, le Royaume-Uni, la Suède, et la Suisse ${ }^{48}$. Elle comprend six catégories de réformes : les réformes du système électoral, les réformes parlementaires, les réformes de fédéralisation et de décentralisation, l'élection directe de la tête de l'exécutif au niveau national ou local, les règlementations sur les référendums et initiatives citoyennes au niveau national, et enfin la régulation de l'accès au suffrage. Cette base de données présente l'intérêt de couvrir un ensemble large (bien que non exhaustif) de réformes des règles démocratiques essentielles. Il est vrai que certaines des institutions identifiées par Lijphart, comme la régulation de la justice constitutionnelle, sont absentes ${ }^{49}$. D'autres, comme la question de la démocratie directe, sont incluses. Pour autant, l'échantillon de réformes reste un des plus larges accessibles, permettant des investigations statistiques sur une série de démocraties consolidées.

Il est à noter que ce qui est comptabilisé comme un cas de réforme est la modification des règles sur l'une des six dimensions incluses. En d'autres termes, si une seule loi modifie à la fois $x$ dimensions de l'architecture institutionnelle, on considère que $x$ réformes ont eu lieu. C'était par exemple le cas lors de la réforme constitutionnelle de 2008 qui incluait à la fois une réforme parlementaire, l'introduction de circonscriptions des français de l'étranger, et des nouvelles règles sur l'organisation de référendums populaires.

\footnotetext{
47 Cf. Appendice 1.

48 Une description exhaustive de la base de données et l'ensemble des réformes prises en compte sont disponibles ici : Camille Bedock, Peter Mair, and Alex Wilson, "Institutional Change in Advanced European Democracies: An Exploratory Assessment," EUI Working Papers RCSAS - EUDO Democracy Observatory, no. 2012/11 (2012), http:/ / cadmus.eui.eu/handle/1814/20817.

${ }^{49}$ Lijphart, Patterns of Democracy. Government Forms and Performance in Thirty-Six Countries, op.cit.
} 


\section{Démêler les déterminants de court et de long terme des réformes}

\section{Les déterminants des réformes à long terme : description des variables}

La première série d'investigations empiriques, qui a pour but de tester les deux premières hypothèses, utilise les 18 démocraties incluses dans la base de données comme unité d'analyse. La variable dépendante utilisée est le nombre de réformes adoptée par pays entre 1990 et 2010, allant de trois (Danemark) à 17 (France). L'analyse consiste principalement en un ensemble de statistiques descriptives (diagrammes de dispersion et régressions linéaires simples) et en une analyse des coefficients de corrélation, du fait du nombre réduit d'observations rendant difficile des analyses plus élaborées comme des régressions linéaires multiples.

Les principales variables analysées concernent d'abord les contraintes institutionnelles structurant chaque système politique, pour vérifier si les systèmes où les règles président aux réformes des institutions sont plus strictes et où le nombre de joueurs de veto au gouvernement est plus élevé réforment moins que les autres. Nous avons ainsi examiné le nombre moyen de joueurs de veto partisans sur la période, mesuré par le nombre moyen de partis présents au gouvernement entre 1990 et 2010 dans chaque pays. La deuxième variable institutionnelle concerne le niveau de rigidité constitutionnelle mesuré sur une échelle allant de 1 à 4 , du moins au plus rigide ${ }^{50}$. La distinction faite ici entre ce que Tsebelis appelle les joueurs de veto institutionnels et partisans se justifie par des recherches menées sur des indices synthétiques montrant que les indices qui mêlent caractéristiques institutionnelles structurelles et caractéristiques dérivant de la compétition tendent à mesurer des concepts différents ${ }^{51}$. Les mesures sélectionnées ici sont aussi parcimonieuses que possible. Le choix de se focaliser sur la rigidité constitutionnelle se justifie ici par le fait que l'on s'intéresse aux obstacles que la constitution place sur le chemin des réformateurs. A propos des joueurs de veto partisans, Roller a montré que les mesures les plus simples (nombre de partis au gouvernement, non pondéré) sont aussi performantes que des mesures plus complexes ${ }^{52}$.

Une des variables qui nous intéresse le plus ici est la mesure du niveau moyen de soutien politique enregistré dans chaque pays entre 1990 et 2010. Nous avons construit une échelle de soutien politique qui s'étend théoriquement entre 0 et $100 \%$, synthétisant un ensemble de mesures: le niveau de confiance envers les partis politiques, envers le parlement, le gouvernement, et le niveau de satisfaction avec la manière dont fonctionne la démocratie dans son pays. L'échelle fait la moyenne des répondants ayant déclaré qu'ils faisant confiance aux institutions citées ci-dessus «assez » ou «beaucoup», et de ceux qui sont «assez » ou "très » satisfaits du fonctionnement de la démocratie, en utilisant les données de l'Eurobaromètre pour les pays appartenant à l'Union européenne et celles des World et des European Value Surveys pour l'Islande, la Norvège et la Suisse. Ce niveau de soutien varie entre $28.6 \%$ pour l'Italie et $63.2 \%$ pour le Danemark.

Enfin, pour vérifier l'impact de la volatilité et de l'alternance à long-terme, on s'intéresse ici au niveau moyen de volatilité totale enregistré sur la période 1990-2010, défini par Bartolini et Mair comme la mesure du changement électoral net entre deux élections consécutives ${ }^{53}$, et

\footnotetext{
${ }^{50}$ Cf. Annexe 2.

51 Edeltraud Roller, The Performance of Democracies: Political Institutions and Public Policies, Oxford, Oxford University Press, 2005.

52 Edeltraud Roller, "Conceptualizing and Measuring Institutions of Democratic Governance: A Critical Review and Empirical Validation of Veto Player Indexes," dans l'atelier Institutional Theory: Issues of Measurement and Change, présenté à l'ECPR Joint Sessions of Workshops, Université d'Ebimbourg, 2003.

53 Stefano Bartolini et Peter Mair, Identity, Competition and Electoral Availability: The Stabilisation of European Electorates, 1885-1985, Cambridge, Cambridge University Press, 1990, p. 17.
} 
mesuré par l'indice de Pedersen ${ }^{54}$. On s'intéresse également à une éventuelle relation à long-terme entre la proportion d'élections sur la période 1990-2010 se soldant par une alternance politique et le nombre de réformes. Les pays inclus dans cette base de données varient fortement en termes de population. Les graphiques reportés ci-dessous prennent en compte la taille de la population à partir du nombre d'électeurs enregistrés à la dernière élection.

${ }^{5}$ Mogens N. Pedersen, "The Dynamics of European Party Systems: Changing Patterns of Electoral Volatility," European Journal of Political Research 7 (1), 1979, pp. 1-26. 
Table 1. Analyse des déterminants de long-terme des réformes démocratiques en Europe de l'Ouest, 1990-2010

\begin{tabular}{l|cccccc} 
& $\begin{array}{c}\text { Rigidité } \\
\text { constitutionnelle }\end{array}$ & $\begin{array}{c}\text { Nombre moyen } \\
\text { de partis au } \\
\text { gouvernement }\end{array}$ & Volatilité & $\begin{array}{c}\text { Proportion } \\
\text { d'alternances } \\
\text { politiques }\end{array}$ & $\begin{array}{c}\text { Niveau de } \\
\text { soutien } \\
\text { politique }\end{array}$ & $\begin{array}{c}\text { Nombre de } \\
\text { réformes }\end{array}$ \\
\hline Allemagne & 3 & 2.6 & 8.4 & 28.6 & 37.6 & 6 \\
Autriche & 2 & 2 & 10.7 & 25 & 50.3 & 10 \\
Belgique & 3 & 4.8 & 11.7 & 43 & 41.7 & 15 \\
Danemark & 3 & 2.3 & 11.3 & 28.6 & 63.2 & 3 \\
Espagne & 3 & 1 & 8.8 & 33.3 & 43.4 & 3 \\
Finlande & 2 & 4 & 9.5 & 66.7 & 52.9 & 11 \\
France & 3 & 2.4 & 13.8 & 80 & 34.1 & 17 \\
Grèce & 2 & 1 & 5.9 & 50 & 37.6 & 9 \\
Irlande & 3 & 2.3 & 9.6 & 0 & 43.5 & 6 \\
Islande & 4 & 2.1 & 12.8 & 28.6 & 42.5 & 7 \\
Italie & 2 & 4.9 & 15.4 & 85.8 & 28.6 & 12 \\
Luxembourg & 2 & 2 & 8.5 & 40 & 60.7 & 5 \\
Norvège & 3 & 2.2 & 15.9 & 66.7 & 59 & 4 \\
Pays-Bas & 4 & 2.7 & 17 & 57.1 & 55.6 & 4 \\
Portugal & 2 & 1.1 & 14.9 & 42.9 & 38 & 13 \\
Royaume-Uni & 1 & 1 & 7.8 & 33.3 & 38.1 & 12 \\
Suède & 2 & 2 & 13.4 & 50 & 50 & 6 \\
Suisse & 2 & 3.9 & & 0 & 53 & 4 \\
\hline Source:CCf: & 2 & 7.9 & & & \\
\hline
\end{tabular}

Source : Cf. Annexe 1. 


\section{La fréquence des réformes à long-terme : la centralité du niveau de soutien politique}

Si l'on examine les coefficients de corrélation entre le nombre de réformes et, respectivement, le niveau de rigidité constitutionnelle et le nombre moyen de partis au gouvernement, on constate qu'il existe une relation légèrement négative entre rigidité constitutionnelle et nombre de réformes, et légèrement positive entre le nombre moyen de partis au gouvernement et le nombre de réformes. Cependant, aucune de ces relations n'est statistiquement significative, comme l'illustre par exemple le diagramme de dispersion de la Figure 1. On voit ici que des pays comme la Belgique, caractérisée par une forte rigidité constitutionnelle et une moyenne de presque cinq partis au gouvernement a adopté un nombre très important de réformes (15), quand d'autres pays aux constitutions plus souples et avec des gouvernements moins pléthoriques ont réformé beaucoup moins. On constate également que la très grande majorité des pays concerné n'entre pas dans l'intervalle de confiance de $95 \%$. Ce premier résultat important suggère qu'à long-terme, et contrairement aux attentes énoncées par notre première hypothèse, le degré de contrainte institutionnelle, qu'il soit mesuré par la rigidité constitutionnelle ou par le nombre de partis au gouvernement n'est pas un frein aux réformes.

Figure 1. Nombre de réformes des règles démocratiques régressé sur le nombre de partis moyen au gouvernement

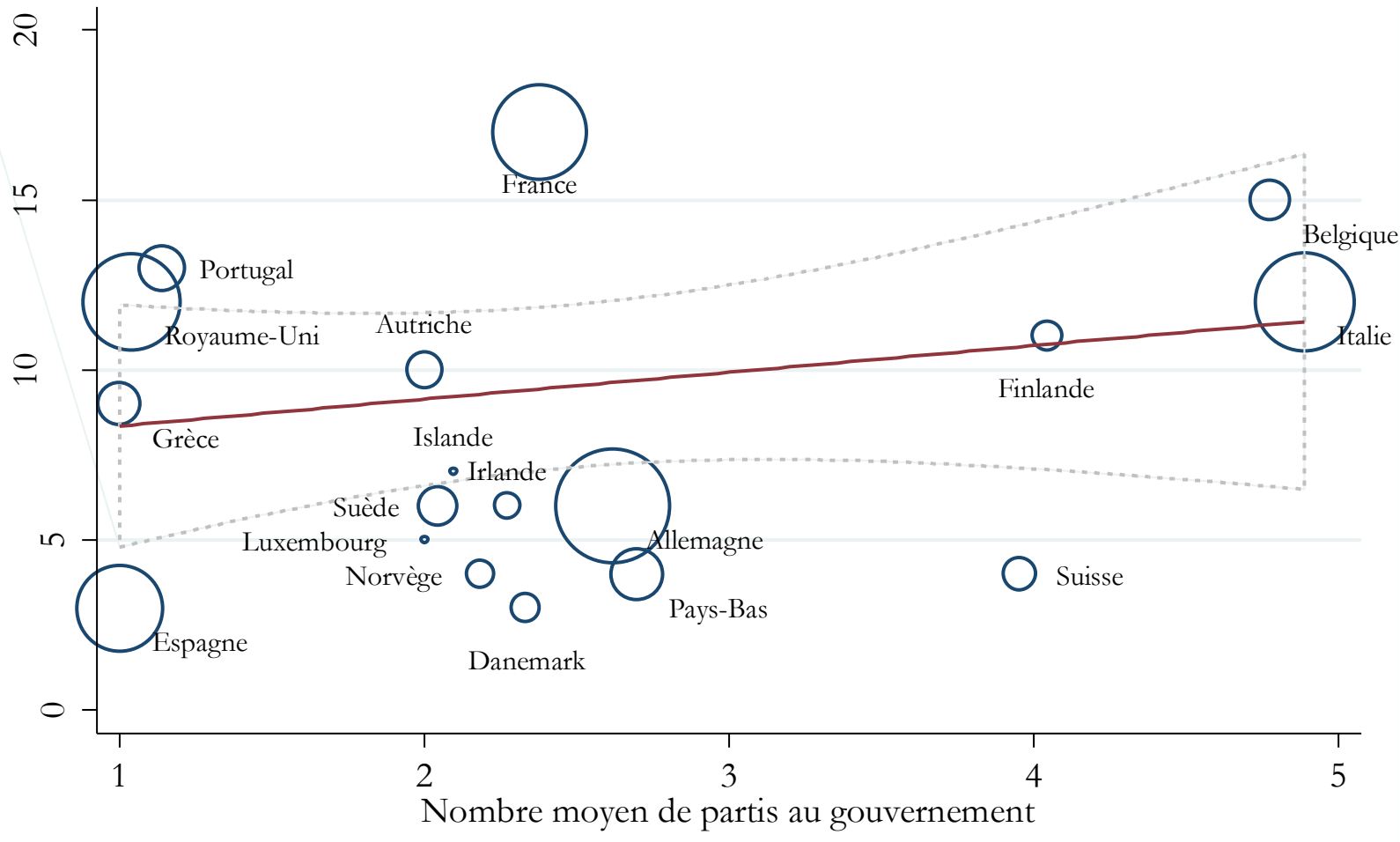

- Pays pondéré par le nombre d'électeurs inscrits

$95 \%$ IC Valeurs prédites

Coefficient de régression : 0.801 (non significatif)

Si on analyse cette fois la relation entre le nombre de réformes adoptées et le niveau de soutien politique, on constate comme attendu une relation négative et statistiquement significative entre les deux variables (corrélation de $-0.66, \mathrm{p}<0.01$ ). Le diagramme de dispersion (Figure 2) de la relation entre le nombre de réformes et le niveau de soutien politique pondéré par le poids de chaque pays montre graphiquement clairement une relation linéaire et négative forte entre les 
deux variables. Plus le niveau de soutien politique est élevé (et donc la confiance envers les institutions et la satisfaction envers la démocratie sont fortes), moins le nombre de réformes démocratiques adoptées est important sur la période 1990-2010. Ainsi, en Italie, où le niveau de soutien politique est le plus bas $(28,1 \%)$, la droite de régression prévoit l'adoption de 13 réformes, tandis qu'au Danemark, où le niveau de soutien politique est le plus élevé (63.2\%), elle indique l'adoption de deux réformes (trois en réalité). La relation n'est bien sûr pas parfaite : on constate en effet que certains pays se situent en dehors de l'intervalle de confiance de $95 \%$, comme la France où le nombre de réformes adoptées est plus élevé que prévu par la droite de régression, ou encore en Allemagne et en Espagne où ce nombre est plus faible que prévu.

Figure 2. Nombre de réformes des règles démocratiques régressé sur le niveau de soutien politique

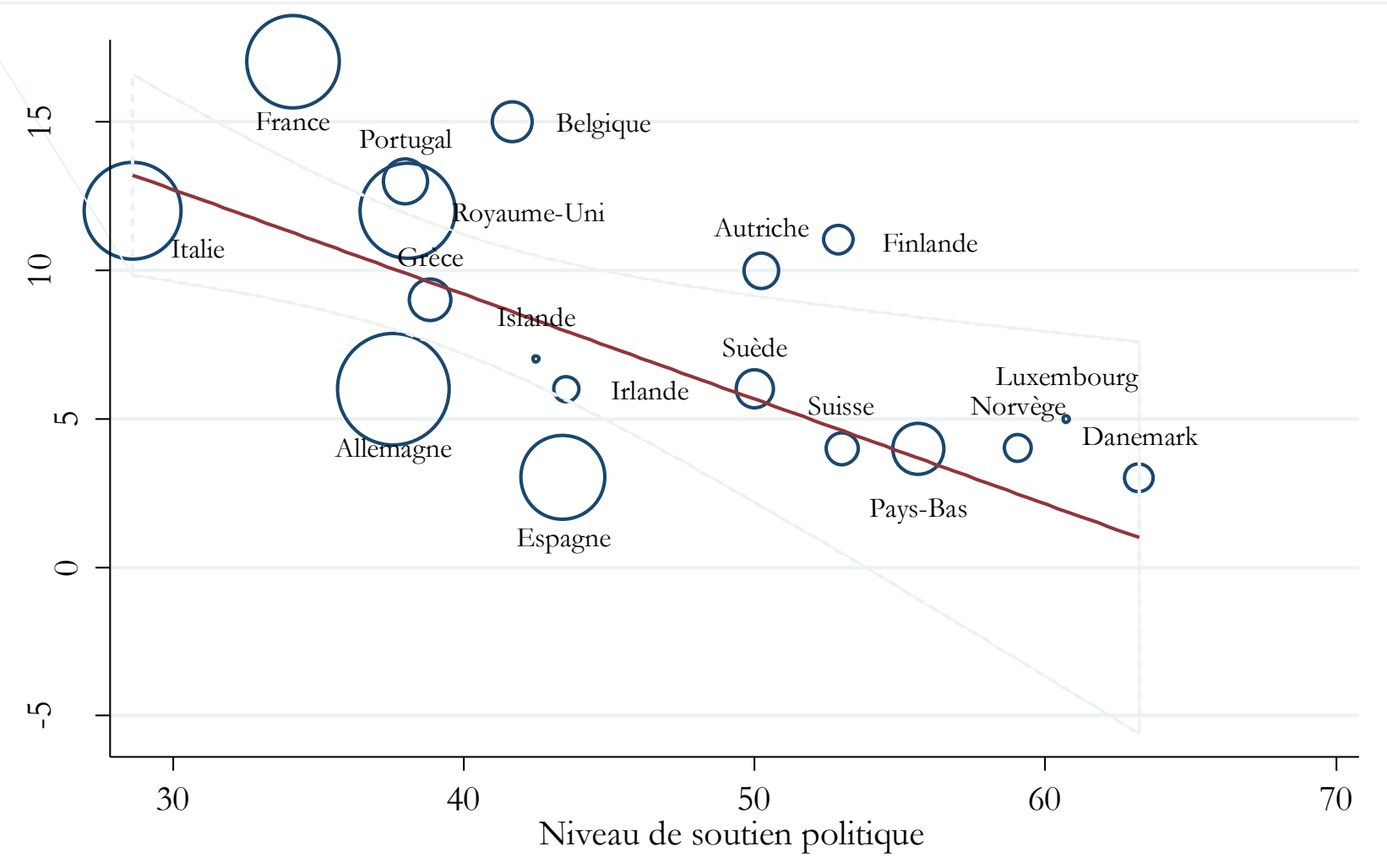

- $\quad$ Pays pondéré par le nombre d'électeurs inscrits

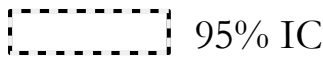

Valeurs prédites

Coefficient de régression : -0.289, p. $<0.01 * * *$

Ainsi, ces analyses descriptives semblent confirmer clairement la deuxième hypothèse, suggérant donc que les pays où le niveau de soutien politique est plus faible ont effectivement plus tendance à réformer leurs règles démocratiques que les autres.

Comme attendu, il n'ya aucune relation statistique significative entre le niveau structurel de volatilité sur la période et le nombre de réformes adoptées. En revanche, on constate une relation positive et statistiquement légèrement significative entre la proportion en pourcentage de législatures qui ont connu une alternance politique et le nombre de réformes adoptées (corrélation de 0.44, p. $<0.1$ ). On constate ainsi que si aucune des législatures de la période 1990 2010 ne s'est soldée par une alternance au pouvoir, le nombre de réformes attendues est de 4 tandis que si l'intégralité des législatures se sont soldées par une alternance politique, la droite de régression indique l'adoption de près de 12 réformes. Reste donc à vérifier si, à court terme, une 
alternance politique en début de législature tend effectivement à favoriser l'adoption de plus de réformes institutionnelles.

Figure 3. Nombre de réformes des règles démocratiques régressé sur le pourcentage de législatures avec alternance

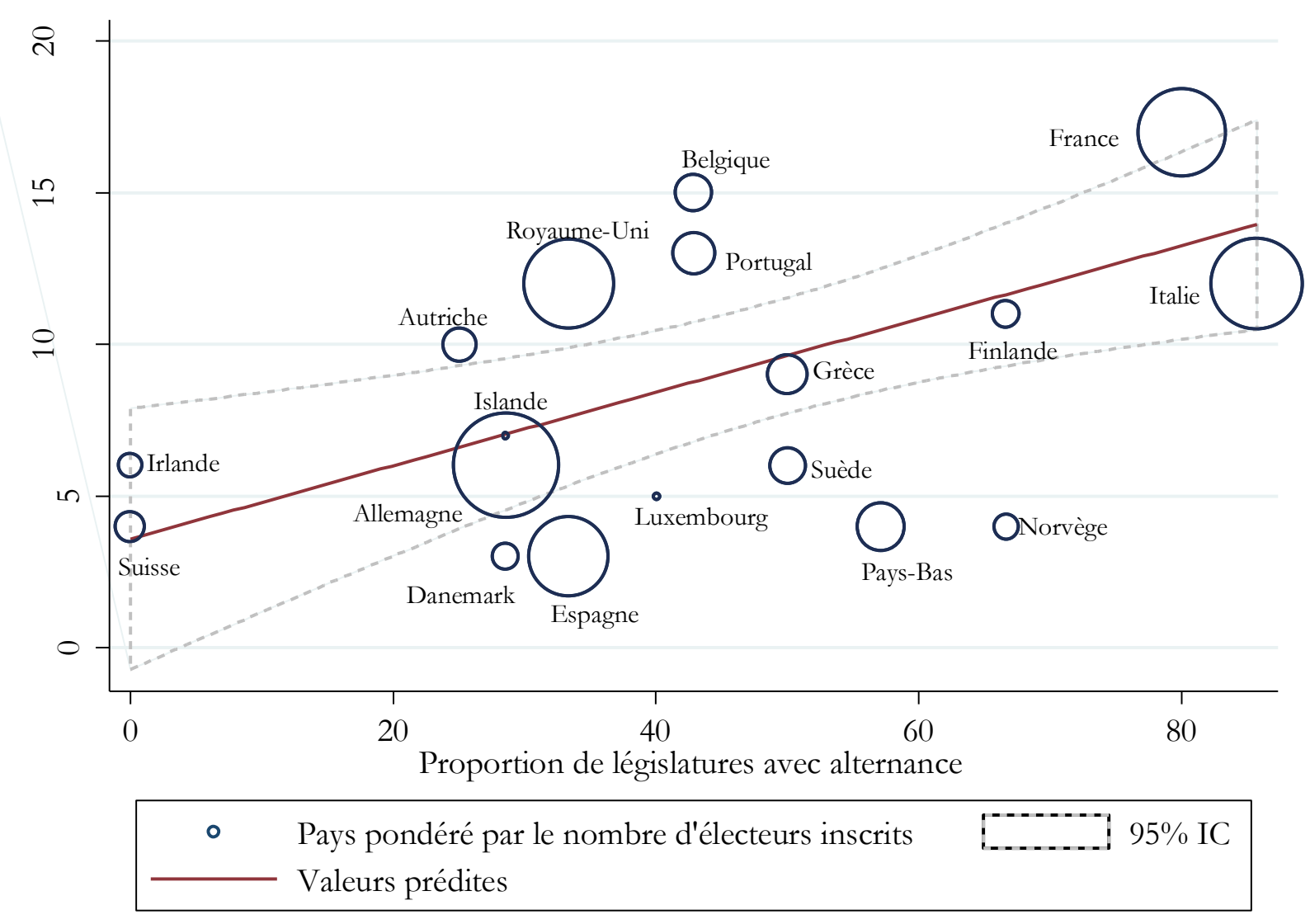

Coefficient de régression : 0.082 , p. $<0.1 *$

\section{Les déterminants des réformes à court-terme : variables et modèles}

Pour tester la troisième et la quatrième hypothèse, l'unité d'analyse retenue est celle de la législature, et la variable dépendante le nombre de réformes adopté dans une législature donnée. Ce nombre varie entre zéro et 7 , avec près de deux tiers $(63 \%)$ des législatures dans lesquelles au moins une réforme institutionnelle est adoptée. On a privilégié cette variable plutôt, par exemple, qu'une variable indiquant seulement s'il y a eu réforme ou non, afin d'avoir une mesure beaucoup plus sensible, moins influencée par d'éventuels effets de seuil arbitraires. La première difficulté méthodologique vient du fait qu'il s'agisse d'une variable dépendante discrète de type modèle de comptage dont la distribution n'est pas normale. 
Table 2. Nombre de réformes adoptées par législature, 1990-2010

Réformes par legislature $\quad \mathrm{N} \quad \%$

\begin{tabular}{c|c|c}
\hline 0 & 43 & 37.1 \\
1 & 37 & 31.9 \\
2 & 18 & 16.4 \\
3 & 9 & 6.9 \\
4 & 3 & 2.6 \\
5 & 2 & 1.7 \\
6 & 3 & 2.6 \\
7 & 1 & 0.9 \\
\hline Total & $\mathbf{1 1 6}$ & $\mathbf{1 0 0 \%}$ \\
\hline \hline
\end{tabular}

Source: base de données SIEPOL, ibid.

Pour vérifier l'éventuelle existence d'un impact à court-terme de la variation du soutien politique, il faudrait idéalement reproduire l'échelle développée plus haut. Cependant, seule la variable portant sur la satisfaction envers le fonctionnement de la démocratie permet une telle analyse au niveau de la législature. On inclut donc dans les modèles suivants l'évolution en pourcentage du niveau agrégé de répondants qui se déclarent «très » ou «assez » satisfaits du fonctionnement de leur démocratie. La deuxième variable explicative importante est l'évolution en pourcentage du niveau total de volatilité d'une élection à l'autre. La troisième et dernière variable explicative importante est l'existence ou non d'une alternance politique en début de législature, définie comme l'existence concomitante d'un changement de l'orientation politique du gouvernement ${ }^{55}$ et d'un changement à la tête de l'exécutif.

Enfin, un certain nombre de variables de contrôle sont introduites. Tout d'abord le nombre de joueurs de veto au gouvernement, cette fois par législature. On contrôle également par le niveau de rigidité constitutionnelle. La troisième variable de contrôle est l'orientation idéologique des gouvernements au pouvoir (divisée en trois catégories : gauche et centre-gauche, droit et centre-droit, grande coalition). Enfin, pour contrôler l'éventuel impact de la conjoncture économique, on introduit dans le modèle le niveau de croissance économique moyen enregistré par législature.

Cette deuxième partie de l'analyse est conduite en utilisant la méthode de la régression négative binomiale, qui est une sous-catégorie des modèles de données de comptage. De tels modèles sont utilisés quand la variable dépendante décompte le nombre d'occurrences d'un évènement sur une période donnée, et consiste donc en une variable discrète composée de nombre positifs et entiers, avec un nombre élevé de zéros et de petites valeurs. Ces modèles permettent de généraliser les modèles linéaires traditionnels qui, non corrigés, mèneraient à des estimateurs biaisés, du fait d'une distribution non normale. On a privilégié la régression binomiale négative à une régression de poisson, à cause du problème rencontré ici de sur-dispersion des données $^{56}$. Deuxièmement, les modèles présentés ci-dessous corrigent les erreurs standards par la méthode des grappes (clusters) afin de tenir compte du problème des erreurs qui ne sont pas distribuées de manière indépendante. Ici, on constate que les observations au sein de chaque groupe (les 18 pays constituant les clusters) sont corrélées, ce qui poserait un problème d'erreurs standards biaisées, et donc d'inférence en l'absence de correction. Par ailleurs, on préfère la correction par la méthode des grappes aux erreurs standards robustes qui nécessitent

\footnotetext{
${ }_{55}$ Cf. Annexe 3.

${ }^{56}$ Joseph M. Hilbe, Negative Binomial Regression, Cambridge, Cambridge University Press, 2011, p. 2. Ici, les données ne remplissent pas le prérequis d'égalité entre la moyenne conditionnelle et la variance, nécessaire pour pouvoir utiliser une régression de Poisson. Le choix de la régression négative binomiale permet dans notre analyse, par rapport à la régression de Poisson, d'ajouter un paramètre qui modélise la surdispersion, et permet donc d'obtenir des intervalles de confiance fiables.
} 
normalement au moins 50 groupes pour être fiables. La qualité des estimations est évaluée grâce au test de Wald, un modèle de vraisemblance qui compare la qualité de l'ajustement du modèle statistique avec un modèle nul où tous les paramètres sont à zéro.

\section{La fréquence des réformes à court-terme : la centralité des bouleversements dans l'arène électorale}

Les résultats sont reportés à partir du rapport du taux d'incidence pour des raisons d'interprétation ${ }^{57}$, avec l'intervalle de confiance de $95 \%$ pour évaluer l'incertitude des coefficients. Le premier modèle n'inclut que les variables de contrôle. Il montre que la rigidité constitutionnelle a un impact négatif et statistiquement significatif sur le nombre de réformes adoptées dans la législature, tandis que le nombre de joueurs de véto présents au gouvernement a, toutes les autres variables étant tenues constantes, un effet positif et statistiquement significatif sur le nombre de réformes adoptées. La croissance économique et l'orientation politique du gouvernement n'apparaissent pas comme ayant un impact statistiquement significatif sur la fréquence des réformes. Si la relation entre rigidité constitutionnelle et nombre de réformes va dans le sens attendu (plus de contrainte limitant à court-terme le nombre de réformes), le lien positif entre nombre de partis au gouvernement et réformes adoptées est en revanche contreintuitif et s'inscrit à nouveau en contradiction avec notre première hypothèse. Le lien entre contrainte institutionnelle et fréquence des réformes démocratiques semble donc obéir à des logiques bien plus complexes qu'une simple relation négative et linéaire. Le deuxième modèle teste l'effet de l'évolution du niveau moyen de satisfaction envers la démocratie sur le nombre de réformes adoptées par législature. Comme suggéré dans la deuxième hypothèse, le niveau de soutien politique a un effet à long-terme, mais pas à court-terme, sur la fréquence des réformes, puisqu'on ne constate pas de relation statistiquement significative entre les évolutions de satisfaction envers la démocratie et le nombre de réformes adoptées. Le troisième modèle montre que, comme attendu dans l'hypothèse 3, la montée de la volatilité a un impact positif et statistiquement significatif sur la fréquence des réformes dans une législature donnée : un point de pourcentage de volatilité supplémentaire est associé à une augmentation de 3\% du nombre de réformes. Pour une interprétation plus lisible des résultats, on a reporté graphiquement le nombre prévu de réformes selon l'évolution du niveau de volatilité. On constate ainsi que dans les cas où la volatilité diminue de $80 \%$ d'une élection à l'autre, le modèle prédit que 0.9 réformes seront adoptées, tandis que si ce niveau est multiplié par trois $(+200 \%)$, ce nombre s'élève à 2.2. Au-delà d'une augmentation de $200 \%$, l'intervalle de confiance devient beaucoup plus large, signifiant que la relation entre volatilité et nombre de réformes est moins claire, faute d'observations en nombre suffisant. Le quatrième modèle s'intéresse à l'effet de l'alternance au pouvoir, et confirme la quatrième et dernière hypothèse. En effet, lorsque toutes les autres variables sont tenues à leur moyenne, le nombre prédit de réformes est de 0.9 en l'absence d'alternance en début de législature, et de 1.7 si l'alternance a lieu, soit presque deux fois plus.

Le cinquième modèle inclut à la fois la variable de l'évolution de la volatilité et celle de l'alternance politique. De nouveau, l'effet des deux variables est statistiquement significatif, alors que, comme dans le modèle précédent, l'effet positif du nombre de joueurs de véto au gouvernement sur le nombre de réformes a disparu. Enfin, le dernier modèle qui inclut l'ensemble des variables analysées confirme à nouveau l'effet de la montée de la volatilité et de l'alternance, avec un nombre prévu de réformes très similaires à ceux déjà exposés. Des investigations supplémentaires non reportées démontrent que l'effet de l'alternance n'est pas conditionnel au niveau de volatilité, ou inversement que l'effet de la volatilité n'est pas

57 Etant donné que la régression binomiale négative est une généralisation du modèle linéaire, ses coefficients ne sont pas directement interprétables. Ici, si les rapports de taux d'incidence sont supérieurs à 1 , on attend que le nombre de réformes augmente et s'il est inférieur à 1 , qu'il diminue. 
conditionnel à la présence ou l'absence d'alternance au pouvoir. Nos modèles semblent converger pour montrer que la montée de la volatilité et l'arrivée de nouveaux acteurs au pouvoir ont un effet indépendant et positif sur le nombre de réformes adoptées dans une législature donnée.

Table 3. Déterminants du nombre de réformes démocratique adoptées par législature en Europe de l'Ouest, 1990-2010

\begin{tabular}{|c|c|c|c|c|c|c|}
\hline & Modèle 1 & Modèle 2 & Modèle 3 & Modèle 4 & Modèle 5 & Modèle 6 \\
\hline $\begin{array}{l}\text { Evolution du niveau de satisfaction } \\
\text { envers la démocratie en } \%\end{array}$ & & $\begin{array}{c}1.006 \\
(0.99-1.02)\end{array}$ & & & & $\begin{array}{c}1.005 \\
(0.99-1.01)\end{array}$ \\
\hline Evolution de la volatilité totale en $\%$ & & & $\begin{array}{c}1.003 * * \\
(1.00-1.01)\end{array}$ & & $\begin{array}{c}1.002 * \\
(1.00-1.01)\end{array}$ & $\begin{array}{c}1.003 * * \\
(1.00-1.01)\end{array}$ \\
\hline Alternance au gouvernement & & & & $\begin{array}{c}1.876 * * * \\
(1.46-2.41)\end{array}$ & $\begin{array}{c}1.708 * * * \\
(1.34-2.18)\end{array}$ & $\begin{array}{l}1.567 * * * \\
(1.14-2.15)\end{array}$ \\
\hline Degré de rigidité constitutionnelle & $\begin{array}{c}0.753 * * \\
(0.60-0.94)\end{array}$ & $\begin{array}{c}0.762 * * \\
(0.60-0.98)\end{array}$ & $\begin{array}{c}0.754 * * \\
(0.58-0.97)\end{array}$ & $\begin{array}{c}0.776^{* *} \\
(0.61-0.99)\end{array}$ & $\begin{array}{c}0.774 * \\
(0.60-1.00)\end{array}$ & $\begin{array}{c}0.787 * \\
(0.60-1.04)\end{array}$ \\
\hline Nombre de partis au gouvernement & $\begin{array}{c}1.138 * * \\
(1.01-1.28)\end{array}$ & $\begin{array}{c}1.103 * \\
(1.00-1.22)\end{array}$ & $\begin{array}{c}1.126 * * \\
(1.01-1.25)\end{array}$ & $\begin{array}{c}1.079 \\
(0.64-1.22)\end{array}$ & $\begin{array}{c}1.081 \\
(0.96-1.21)\end{array}$ & $\begin{array}{c}1.056 \\
(0.96-1.17)\end{array}$ \\
\hline
\end{tabular}

Orientation idéologique

(cat. de réf: gauche/ centre-gauche)

Gouvernement de droite/ centre-droit

\begin{tabular}{lcccccc} 
Gouvernement de droite/ centre-droit & 1.221 & 1.221 & 1.232 & 1.310 & 1.308 & 1.281 \\
& $(0.76-1.98)$ & $(0.74-2.00)$ & $(0.76-1.99)$ & $(0.82-2.09)$ & $(0.82-2.09)$ & $(0.79-2.07)$ \\
Gowvernement de grande coalition & 0.838 & 0.820 & 0.862 & 0.962 & 0.966 & 0.930 \\
& $(0.48-1.45)$ & $(0.45-1.49)$ & $(0.51-1.46)$ & $(0.58-1.59)$ & $(0.58-1.60)$ & $(0.52-1.65)$ \\
Croissance & 1.074 & 1.053 & $\mathbf{1 . 0 8 3} *$ & 1.083 & $\mathbf{1 . 0 8 8} *$ & 1.073 \\
& $(0.98-1.16)$ & $(0.95-1.17)$ & $\mathbf{( 0 . 9 9 - 1 . 1 8 )}$ & $(0.98-1.19)$ & $\mathbf{( 0 . 9 9 - 1 . 1 9 )}$ & $(0.97-1.19)$ \\
\hline Observations & 116 & 110 & 116 & 116 & 116 & 110 \\
Nombre de clusters & 18 & 18 & 18 & 18 & 18 & 18 \\
& & & & & & \\
Alpha & 0.468 & 0.440 & 0.394 & 0.352 & 0.325 & 0.306 \\
& $(0.25-0.86)$ & $(0.24-0.80)$ & $(0.19-0.84)$ & $(0.15-0.82)$ & $(0.13-0.84)$ & $(0.12-0.78)$ \\
Pseudo R ${ }^{2}$ de MacFadden's & 0.02 & 0.03 & 0.03 & 0.05 & 0.05 & 0.05 \\
Pseudo R de Cox-Snell Pseudo & 0.06 & 0.07 & 0.10 & 0.13 & 0.15 & 0.15 \\
Chi2 de Wald & 17.63 & 20.44 & 14.18 & 30.08 & 31.89 & 42.53 \\
Prob $>$ chi2 & 0.004 & 0.002 & 0.027 & 0.000 & 0.000 & 0.000 \\
\hline
\end{tabular}

Gouvernement de grande coalition

0.838

0.820

(0.64-1.22)

$(0.96-1.17)$

Note: Les coefficients sont reportés en termes de ratios de taux d'incidence. Les intervalles de confiance de $95 \%$ sont entre parenthèse. La variable dépendante est le nombre total de réformes adoptées par législature entre 1990 et $2010 .{ }^{*} \mathrm{p}<0.1 * * \mathrm{p}$ $<0.05^{* * *} \mathrm{p}<0.01$ 
Figure 4. Nombre prédit de réformes selon l'évolution de la volatilité totale (modèle 3)

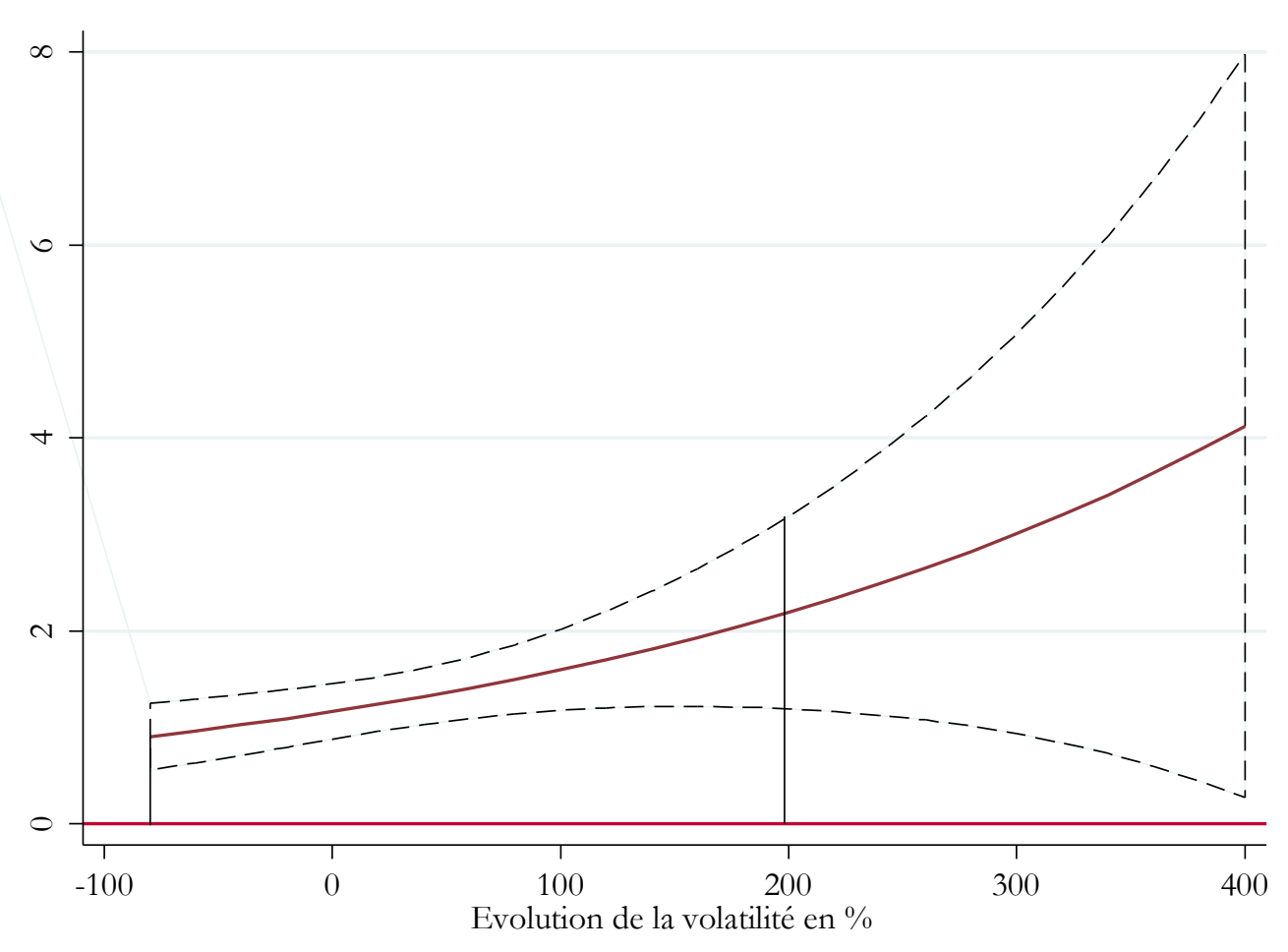

Note: les valeurs de l'évolution de la volatilité sont comprises entre une baisse de $77 \%$ et une augmentation de $357 \%$

D'un point de vue substantiel, ces modèles nous permettent d'atteindre plusieurs conclusions. Premièrement, ces modèles confirment que la logique institutionnelle qui voudrait que ce qui explique principalement la fréquence des réformes démocratiques soit le degré de contrainte institutionnelle qui pèse sur les acteurs doit être fortement relativisée. En effet, si la rigidité constitutionnelle semble bien avoir un impact négatif sur le nombre de réformes adoptées par législature, les autres variables étant tenues constantes, on constate en revanche qu'à courtterme, il semble que la présence au gouvernement d'un nombre important de partis ne soit pas un frein à l'adoption de réformes plus nombreuses, mais possiblement un accélérateur. La logique politique président aux réformes est en revanche clairement confirmée par les modèles empiriques. On constate ici que les élites sont très sensibles aux évènements se déroulant dans l'arène électorale quand elles décident de promouvoir des réformes institutionnelles. La montée de la volatilité peut être pensée comme une manifestation tangible de changements électoraux qui a pour conséquence une montée de l'incertitude pour les partis politiques. L'alternance, en amenant au pouvoir des partis avec des préférences différentes de celles des sortants quant aux réformes démocratiques, donne une fenêtre d'opportunité à des partis pour mettre en œuvre des réformes.

\section{Conclusion}

Cette étude s'est attachée à montrer que la logique qui préside aux réformes démocratiques est, avant tout, une logique politique. La logique institutionnelle qui voudrait que la fréquence des réformes s'explique avant tout par le degré de contrainte institutionnelle présente dans chaque système se révèle ici avoir une portée explicative très limitée. Si une plus forte 
rigidité constitutionnelle tend à limiter le nombre de réformes à court-terme, elle n'a pas d'impact à long-terme sur le nombre de réformes adoptées. De façon plus surprenante encore, la présence de multiples partis au gouvernement n'apparaît pas comme étant un frein aux réformes, ni à longterme, ni à court-terme. Nos résultats montrent donc qu'il n'existe pas de relation linéaire entre contrainte institutionnelle et fréquence des réformes, un résultat qui appelle à examiner de près des processus de réformes démocratiques pour comprendre quand et comment les contraintes institutionnelles structurent les débats et l'issue finale des réformes démocratiques. En effet, des études de cas portant notamment sur l'Italie et les réformes électorale et constitutionnelle de 2005 ont déjà eu l'occasion de montrer qu'en présence de vastes coalitions, la capacité à parvenir à un accord sur des réformes institutionnelles dépend de la capacité à négocier un compromis donnant des garanties à l'ensemble des acteurs de la coalition ${ }^{58}$. On retrouve dans cet exemple l'idée de «consensus ambigu» de Palier postulant qu'un consensus sur des réformes ne fasse pas nécessairement l'objet d'un accord clair sur les objectifs assignés. Cela permettrait d'expliquer pourquoi la présence de multiples joueurs de véto n'est pas toujours à même de bloquer le changement $^{59}$. Deuxièmement, cette étude a montré qu’à long-terme, le niveau de soutien politique est fortement lié au nombre de réformes adoptées. Les démocraties les plus touchées par l' «érosion du soutien politique » diagnostiqué par Dalton, comme l'Italie ou la France sont donc beaucoup plus vulnérables aux réformes démocratiques. Toujours dans le cas de l'Italie, cette explication permet de comprendre pourquoi la question des réformes institutionnelles est aussi prégnante en 2014 qu'en 1993. A court-terme, on a mis en évidence l'impact de la montée de la volatilité et de l'alternance politique sur le nombre de réformes adoptées dans une législature donnée.

Dans un contexte de crise prolongée, on peut donc s'attendre à ce que le contexte soit favorable à une multiplication des réformes démocratiques dans les démocraties consolidées. Le niveau de soutien politique est à la baisse. Les élections irlandaises de 2011, ou celles qui se sont tenues en Italie en 2013 ont enregistré des niveaux de volatilité sans précédent. Dans la plupart des élections qui ont eu lieu depuis la crise de 2009, les partis ou coalitions au pouvoir ont subi de lourdes défaites électorales. Reste encore à mieux comprendre les mécanismes qui lient soutien politique, alternance et volatilité dans le cadre d'études de cas approfondies. Une autre question de recherche directement dérivée des résultats présentés ici vient également immédiatement à l'esprit. Si ce sont les démocraties les plus désavouées et les plus frappées par les bouleversements électoraux qui réforment leur démocratie le plus, ces réformes sont-elles à même d'endiguer la chute du soutien politique et de limiter l'impact des vicissitudes électorales?

(signes : 67 951)

\footnotetext{
${ }^{58}$ Camille Bedock, «Du mattarellum au porcellum : une comparaison des réformes électorales de 1993 et 2005 en Italie, » Pôle Sud no 34 (1), 2011, pp. 27-44; Salvatore Vassallo, "The Constitutional Reforms of the Center-Right," dans Quo Vadis?, Carlo Guarnieri et James L. Newell (ed.), Bologne, Istituto Carlo Catteneo, 2005, pp. 117-35.

${ }^{59}$ Bruno Palier, « De la crise aux réformes de l'État-providence. Le cas français en perspective comparée, » Revue française de sociologie 43 (2), 2002 : pp. 243-75; Bruno Palier, La réforme des retraites, Paris, Presses universitaires de France, 2003.
} 


\title{
Annexes. «Les déterminants politiques de la fréquence des réformes démocratiques en Europe de l'Ouest, 1990-2010 »
}

\author{
Annexe 1. Présentation de la base de données SIEPOL "Institutional Change in Advanced European \\ Democracies 》
}

Dans cet article, les réformes prises en compte ont été comptabilisées à partir de la base de données SIEPOL "Institutional Change in Advanced European Democracies"60, et des six dimensions suivantes de réforme : les réformes du système électoral (1), les réformes parlementaires (2), les réformes fédérales et de décentralisation (3), l'élection directe du chef de l'exécutif au niveau local ou national (4), la régulation des référendums et des initiatives citoyennes au niveau national (5) et la régulation de l'accès au suffrage (6).

L'article de Bedock, Mair et Wilson dresse une liste de réformes, en mentionnant les informations suivantes : le pays dans lequel elles ont été adoptées, l'année d'adoption, la dimension réformée, et de brèves indications sur le contenu de la réforme. Les réformes sont définies de la manière suivante, comme des «changements qui affectent la relation directe entre les élites, les partis, les citoyens, les gouvernements, et les parlements $»^{61}$. La collecte des données a eu lieu en deux étapes. Dans un premier temps, l'équipe du projet SIEPOL a accédé à des sources primaires et secondaires sur les réformes (parmi lesquelles on peut citer l'EJPR Political Data Yearnook, les données sur les autorités régionales de Marks et al., la Comparative Study of Electoral Systems, les données IDEA, etc.). Dans un second temps, les fichiers recueillis pour chacune des 18 démocraties incluses ont été envoyé à des experts nationaux pour vérification et correction. La liste complète de réformes est accessible avec le lien suivant: http://cadmus.eui.eu/bitstream/handle/1814/20817/RSCAS 2012 11.pdf?sequence=1

Quelques exemples sont mentionnés ci-dessous.

\section{1- Réformes du système électoral}

Il s'agit ici des réformes qui modifient la formule électorale, le système transformant les voix en sièges, et les modalités d'expression du suffrage. Ces réformes concernent aussi bien la modification ou la substitution d'une formule électorale que la modification des frontières des circonscriptions ou l'introduction d'un seuil légal pour entrer au Parlement.

On peut citer l'exemple de la modification des frontières des circonscriptions électorales françaises en 2009, ou la substitution d'un système électoral majoritaire mixte par un système électoral proportionnel avec bonus en Italie en 2005.

\section{2- Réformes parlementaires}

Cette catégorie inclut les réformes qui altèrent la composition du parlement, les règles législatives essentielles qui régulent son fonctionnement, le nombre de chambres, la relation entre le pouvoir exécutif et le Parlement, et la durée des mandats au niveau national.

Les réformes prises en compte dans cette catégorie incluent par exemple l'introduction d'une chambre unique pour remplacer le Parlement tricaméral qui existait auparavant en Islande en 1991, la réduction du nombre de députés mise en place au Portugal en 1997, ou la réforme qui accroît le pouvoir du Parlement face au président en Finlande adoptée en 2000.

\section{3- Décentralisation et réformes fédérales}

Cette catégorie de réforme comprend les modifications de l'équilibre des pouvoirs entre le niveau national et les régions ou entités fédérées en termes de compétences, d'autonomie fiscale, ou d'instruments de

\footnotetext{
${ }^{60}$ Bedock, Mair, et Wilson, "Institutional Change in Advanced European Democracies.", op. cit.

${ }^{61}$ Ibid., p. 3.
} 
contrôle, ainsi que les normes régulant le nombre et les modalités d'organisation des collectivités territoriales.

Cette dimension comprend par exemple la réforme de la Loi fondamentale allemande pour donner aux Länder la capacité de s'impliquer directement dans le processus de décision européen en 1992, ou la modification des règles de financement des collectivités territoriales permettant aux régions espagnols de lever leurs propres impôts en 1996.

\section{4- Election directe des chefs de l'exécutif an niveau national ou local}

Cette catégorie inclut les réformes permettant l'élection directe du responsable du pouvoir exécutif au niveau national, municipal, régional ou provincial, adopté par le parlement national et s'appliquant à l'intégralité ou partie du territoire.

On peut citer l'introduction de l'élection directe du président en Finlande par un système à deux-tours adoptée en 1994, ou encore la série de réformes régulant l'élection directe des maires, des présidents de régions et de provinces adoptées en Italie entre 1993 et 1999.

\section{5- Régulation de la démocratie directe au niveau national}

Cette série de réformes inclut les cas dans lesquels les normes régulant les initiatives citoyennes (contraignantes ou consultatives) où les référendums ont été introduites ou modifiées au niveau national.

Cette cinquième catégorie de réformes inclut ainsi l'autorisation du droit de pétition et des référendums consultatifs locaux et nationaux introduite aux Pays-Bas en 2004, ou l'introduction en 1997 au Portugal d'un amendement constitutionnel autorisant les citoyens à proposer un référendum avec l'accord du parlement.

\section{6- Accès au suffrage}

Cette dernière catégorie de réformes concerne tous les cas dans lesquels le droit de suffrage a été étendu à de nouvelles catégories de la population (étrangers, individus de moins de 21 ans...) ou dans lesquels des méthodes alternatives pour voter ont été introduites ou facilitées, comme le vote postal, le vote électronique, l'introduction de circonscriptions pour les citoyens vivant à l'étranger, etc.

On peut citer l'exemple de l'extension du droit de suffrage aux citoyens hors-UE vivant au Luxembourg pour les élections locales adoptée en 2003 au Luxembourg, ou la réforme facilitant le vote postal et le vote par procuration mise en place en 1999 au Royaume-Uni. 
Annexe 2. Présentation des variables explicatives des déterminants de long-terme des réformes

\begin{tabular}{|c|c|}
\hline Variables & Mesure \\
\hline Soutien politique & $\begin{array}{l}\text { Echelle de } 0 \text { à } 100 \% \text { construite à } \\
\text { partir de } 4 \text { variables, résumant : } \\
\text { - Le pourcentage moyen de } \\
\text { répondants d'un pays donné } \\
\text { qui fait confiance aux partis } \\
\text { politiques, au parlement, et } \\
\text { au gouvernement "assez" ou } \\
\text { "beaucoup" } \\
\text { Le pourcentage moyen de } \\
\text { répondants qui sont "très" } \\
\text { ou "assez" satisfaits du } \\
\text { fonctionnement de la } \\
\text { démocratie dans leur pays }\end{array}$ \\
\hline
\end{tabular}

\section{Source}

Eurobaromètres 31, 33, 34, 35, 36, 37, 38.0, 39.0, 40, 41.0, 42, 43.1, 44.3, 47.1, 48, 49, 51, 52, 53, $54.1,56.2,58.1,59.1,60.1,61,62,62.2,63.4,65.2$, $68.1,72.4,73.4$

Pour l'Islande, la Norvège et la Suisse : Module 1, 2 and 3 de la Comparative Study of Electoral systems parue in 2003, 2007 and 2011, complete par des données du Mannheim Eurobarometer trend file, 1970-2002 pour la Norvège, 1990-1995

Observations pour :

- La confiance envers les partis: Allemagne, Autriche, Belgique, Danemark, Espagne, Finlande, France, Grèce, Irlande, Italie, Luxembourg, PaysBas, Suède, Royaume-Uni : 1997, 99-2010, Islande: 2009

Norvège, Suisse: 1996, 2008

Portugal: 1998-2010

- La confiance envers le parlement: Allemagne, Autriche, Belgique, Danemark, Espagne, Finlande, France, Grèce, Irlande, Italie, Luxembourg, PaysBas, Suède, Royaume-Uni : 1997, 99-2010, Islande: 1990, 1999, 2008

Norvège: 1990, 1996, 2008

Portugal: 1990-2010

Suisse: 1996, 2008

- La confiance envers le gouvernement: Allemagne, Autriche, Belgique, Espagne, Finlande, France, Grèce, Irlande, Italie, Luxembourg, Pays-Bas, Suède, RoyaumeUni : 1997, 99-2010, Danemark: 1997, 1999, 2001-10

Islande: 2010

Norvège Suisse: 1996, 2008

Portugal: 1998-2010

- La satisfaction avec la démocratie: Autriche: 1995, 1997-2007, 2009-10

Belgique, Danemark, Espagne, France, Grèce, Italie, Luxembourg, Pays-Bas, Portugal, Royaume-Uni : 1990-95, 19972007, 2009-10

Finlande: 93-95, 97-2007, 2009-10 


\begin{tabular}{|c|c|c|}
\hline & & $\begin{array}{l}\text { Allemagne: 90-95, 97-2006, 2009-10 } \\
\text { Islande: 1990, 2003, } 2007 \\
\text { Irlande: 1990-2007, 2009-2010 } \\
\text { Suisse: 1999, 2003, } 2007\end{array}$ \\
\hline $\begin{array}{lll}\text { Joueurs } & \text { de } & \text { veto } \\
\text { partisans } & & \\
\end{array}$ & $\begin{array}{l}\text { Nombre moyen de partis présents au } \\
\text { gouvernement, 1990-2010 }\end{array}$ & $\begin{array}{l}\text { Données sur la composition des gouvernements } \\
\text { de la base de données Parlgov } \\
\text { http://www.parlgov.org/stable/data.html }\end{array}$ \\
\hline Volatilité totale $^{a}$ & Volatilité totale moyenne, $1990-2010$ & $\begin{array}{l}\text { Données électorales pour les élections } \\
\text { parlementaires de la base de données Parlgov } \\
\text { http://www.parlgov.org/stable/data.html }\end{array}$ \\
\hline $\begin{array}{l}\text { Proportion } \\
\text { d'alternances } \\
\text { politiques }\end{array}$ & $\begin{array}{l}\text { Proportion de législatures dans } \\
\text { laquelle il y a eu une alternance } \\
\text { politique. L'alternance est définie } \\
\text { comme suit: } \\
\text { présente si le chef de l'exécutif et } \\
\text { l'orientation politique du cabinet } \\
\text { change au début d'une législature, } \\
\text { absente dans les autres cas }\end{array}$ & $\begin{array}{l}\text { Données sur la composition des gouvernements } \\
\text { de la base de données Parlgov } \\
\text { http://www.parlgov.org/stable/data.html }\end{array}$ \\
\hline
\end{tabular}


Annexe 3. Présentation des variables explicatives des déterminants de court-terme des réformes

Variables

Variables explicatives
Mesure

Source

\begin{tabular}{|c|c|c|}
\hline $\begin{array}{c}\text { Evolution de la } \\
\text { satisfaction envers la } \\
\text { démocratie }\end{array}$ & $\begin{array}{l}\text { Evolution en pourcentage d'une } \\
\text { législature à l'autre des répondants } \\
\text { "très" ou "assez" satisfaits du } \\
\text { fonctionnement de la démocratie } \\
\text { dans leur pays } \\
\text { (formulation de la question } \\
\text { originale: "On the whole, are you } \\
\text { very satisfied, fairly satisfied, not very } \\
\text { satisfied or not at all satisfied with } \\
\text { the way democracy works in (YOUR } \\
\text { COUNTRY)?" }\end{array}$ & Cf. Annexe 2 \\
\hline $\begin{array}{l}\text { Evolution de la volatilité } \\
\text { totale }^{\mathrm{a}}\end{array}$ & $\begin{array}{l}\text { Evolution en pourcentage de la } \\
\text { volatilité totale d'une election àl'autre }\end{array}$ & $\begin{array}{l}\text { Données électorales pour les } \\
\text { elections parlementaires de la base de } \\
\text { données Parlgov } \\
\text { http://www.parlgov.org/stable/data } \\
\text {.html }\end{array}$ \\
\hline Alternance politique ${ }^{b}$ & $\begin{array}{l}1 \text { si le chef de l'exécutif et } \\
\text { l'orientation politique du cabinet } \\
\text { change au début d'une législature, } 0 \\
\text { dans les autres cas }\end{array}$ & $\begin{array}{l}\text { Données sur la composition des } \\
\text { gouvernements de la base de } \\
\text { données Parlgov } \\
\text { http://www.parlgov.org/stable/data } \\
. \text { html }\end{array}$ \\
\hline Variables de contrôle & & \\
\hline $\begin{array}{c}\text { Joueurs de veto } \\
\text { partisans }\end{array}$ & $\begin{array}{l}\text { Nombre de partis présents au } \\
\text { gouvernement }\end{array}$ & $\begin{array}{l}\text { Données sur la composition des } \\
\text { gouvernements de la base de } \\
\text { données Parlgov } \\
\text { http://www.parlgov.org/stable/data } \\
\text {.html }\end{array}$ \\
\hline $\begin{array}{c}\text { Rigidité } \\
\text { constitutionnelle }\end{array}$ & Cf. Annexe 2 & Cf. Annexe 2 \\
\hline $\begin{array}{l}\text { Orientation idéologique } \\
\text { de la législature }\end{array}$ & $\begin{array}{l}\text { Classification des législatures en } \\
\text { fonction de l'orientation idéologique } \\
\text { des gouvernements en trois } \\
\text { catégories : gauche/ centre gauche, } \\
\text { droite/ centre-droit, grande coalition. } \\
\text { Les grandes coalitions doivent } \\
\text { inclure tous les principaux partis } \\
\text { politiques de droite et de gauche au } \\
\text { pouvoir. Quand un ou plusieurs de } \\
\text { ces partis sont exclus, et que le } \\
\text { gouvernement est composé de partis } \\
\text { de gauche et de droite, on a regardé } \\
\text { de quel bord était le premier ministre } \\
\text { pour classer le gouvernement à } \\
\text { gauche ou à droite. } \\
\text { Dans les cas où plusieurs }\end{array}$ & $\begin{array}{l}\text { Données sur la composition des } \\
\text { gouvernements de la base de } \\
\text { données Parlgov } \\
\text { http://www.parlgov.org/stable/data } \\
\text {.html }\end{array}$ \\
\hline
\end{tabular}




\begin{tabular}{|c|c|c|}
\hline & $\begin{array}{l}\text { gouvernements se sont succédé dans } \\
\text { la même législature, la classification } \\
\text { dépend du gouvernement qui a duré } \\
\text { le plus longtemps. Les } \\
\text { gouvernements techniques et } \\
\text { intérimaires ont été inclus dans la } \\
\text { catégorie « grande coalition». }\end{array}$ & \\
\hline Croissance & $\begin{array}{l}\text { Pourcentage moyen de croissance } \\
\text { économique du PIB en prix } \\
\text { constants dans une législature } \\
\text { données }\end{array}$ & $\begin{array}{l}\text { Données du Fonds Monétaire } \\
\text { International } \\
\text { www.imf.org }\end{array}$ \\
\hline
\end{tabular}

${ }^{\mathrm{a}}$ La formule est la suivante: Volatilité totale $=|\mathrm{PiV}|+|\mathrm{PjV}| \ldots+|\mathrm{PnV}| / 2 .|\mathrm{PiV}|$ correspond au changement en termes absolus du vote pour le parti I d'une election à l'autre, $|\mathrm{Pj} \mathrm{V}|$ à ce changement pour le parti j, etc. La somme est divisée par 2 pour éviter de compter deux fois gains et pertes.

b La classification des gouvernements n'a pas toujours été simple, notamment dans les pays caractérisé par de larges coalitions (Finlande, Pays-Bas, Italie, Belgique), ou par une vie politique mal définie par le clivage gauche/droite (Irlande). Tous les gouvernements irlandais ont ici été qualifies comme étant de centre-droit. 